\title{
COMPUTER MANUFACTURED PATIENT-SPECIFIC AUTO-REDUCIBLE PLATE VERSUS STANDARD TRAPEZOID PLATE IN UNILATERAL SUBCONDYLAR FRACTURES
}

\author{
Khaled Amr ${ }^{*}$, Heba M. Kamel ${ }^{*}$ and Hussein Hatem **
}

\begin{abstract}
Purpose: Subcondylar fractures account for a high incidence of mandibular fractures representing a frustrating clinical dysfunction and serious complications when maltreated. This study aimed to compare the computer designed and manufactured patient-specific auto-reducible plate to the standard trapezoid plate in attempt to reach the optimal geometrical design for subcondylar fracture plate fixation.
\end{abstract}

Patients and methods: A total of sixteen patients suffering from unilateral subcondylar fracture were included in this study from the outpatient clinic of the department of oral and maxillofacial surgery, faculty of Dentistry, Cairo University. All patients were subjected to preoperative clinical evaluation and 3-D CT examination to assess the degree of fracture displacement and condylar location. The selected patients were divided into two groups, eight patients each. In group A patients, virtual fracture reduction through mirroring of the unaffected intact side was performed, which was then used as a template for designing and processing of the titanium patient-specific condylar plate via direct metal laser sintering. While in group $B$ patients, the unilateral sub-condylar fractures were manually reduced into position and fixed in place by the standard trapezoidal mini-plate. Fixation of the plates in both groups was then performed using 2.0 mini-screws. Postoperative clinical assessment of the occlusion, Maximum inter-incisal opening (MIO) and mandibular deviation was done at one week, 1 month \& 3 months. Immediate postoperative radiographic assessment was performed through the superimposition of the postoperative CT data of the fixed fracture on the preoperative virtually reduced mandibular model as a reference CT data to validate and compare the accuracy of the achieved postoperative fixation in both groups.

Results: The surgeries in all cases proceeded without any complications. Surgical site examination was normal with no signs of infection. The occlusion was satisfactory at the end of the study interval despite the initial slight immediate postoperative occlusal discrepancy found in both groups. Deviation was not completely absent in both groups at the end of the follow-up period. The MIO ranged from 39-47 $\mathrm{mm}$ at the end of the study with no significant difference between both groups. The CT scan revealed the almost precise adaptation of both plates in place with no significant difference, however, with the advantageous fracture auto-reduction, intra-operative time saving and ease of application recorded with the use of the PS-condylar plate in group A patients. The mean operating time for reduction and fixation using the PS-condylar plate was 90 minutes compared to a mean of 88 minutes using the standard reduction and trapezoid plate fixation technique.

* Lecturer of Oral and Maxillofacial Surgery, Oral and Maxillofacial Surgery Department, Faculty of Dentistry, Cairo University.

** Associate Professor of Oral and Maxillofacial Surgery, Oral and Maxillofacial Surgery Department, Faculty of Dentistry, Cairo University. 
Conclusion: Despite of the approaching accuracy of both plates, preoperative digital virtual planning and PS-plate processing were highly beneficial in the auto-reduction of the fractured segments. The intra-operative time saving and ease of application with less obtained surgical errors due to the planned surgery and the perfect fit of the prosthesis were found very valuable. Therefore, accommodating with the continuous technological updates, the integration of computer programs in surgery planning and plate designing and manufacturing in subcondylar fractures is highly desirable due to its great benefits.

KEYWORDS: Subcondylar fracture, Computer-manufactured plate, auto-reducible.

\section{INTRODUCTION}

Mandibular subcondylar fractures, one of the most controversial fractures concerning diagnosis and treatment, represent mainly $25-35 \%$ of all mandibular fractures ${ }^{(1-3)}$.

The reported management of subcondylar fractures included a period of Maxillo-mandibular fixation (MMF), functional therapy or open reduction and internal fixation (ORIF). Although choosing the optimal treatment method is still under debate, the absolute indications and advantages of ORIF of the fractured segments with the use of titanium plates and screws remains constant by most surgeons ${ }^{(4-5)}$.

Valiati et al ${ }^{(5)}$ declared the better occlusal results, anatomic restoration and faster recovery rates with open reduction than with non-surgical techniques, however with the similar functional results for both groups.

There are many techniques which provide fixation of the subcondylar fractures including intraosseous wiring, lag-screw and plates. There are many types of plates including mini-plates, mini-dynamic compression plates, microplates and resorbable plates. Authors recommended the use of miniplates when ORIF is utilized regardless of its geometric shape ${ }^{(6)}$.

Choi et al (7) affirmed in their study that 2-miniplates fixation in different planes is the method of choice in fixation of subcondylar fractures to achieve a 3-dimensional fixation form.

Accordingly, trapezoidal condylar plate (TCP) was used as a 3D plate designed for adaptation in the anatomically constricted condylar neck; hence, fulfilling the criteria of 2 single miniplates with reduced hardware ${ }^{(8)}$.

Recent studies used computer programs in planning of complex cranio-maxillofacial surgeries to attain higher accuracy and reduction of the complications ${ }^{(9)}$.

Computer-assisted preoperative planning enables the surgeon to use the CT data to form a 3D template of the skull for visualization and diagnosis. Together with mirroring and creation of a custom plate, surgical simulation provided a guide for precise and safe placement and fixation of the plates $^{(10)}$.

Accordingly, a 3-D trapezoidal patient-specific PS-plate was designed and manufactured in which an extended wing on the posterior border of the condylar neck was added to incase the two fractured segments allowing their auto-reduction.

This study presents the distinction in the clinical application of the PS-Auto-reducible plate and the standard trapezoidal plate, in attempt to reach the optimal geometrical design for subcondylar fracture mini-plate fixation.

\section{PATIENTS AND METHODS}

A total of sixteen patients were included in this study, from the outpatient clinic of the department of oral and maxillofacial surgery, faculty of Dentistry, Cairo University. They were 13 males and 3 females with a mean age of 42 years. 
They all had a unilateral sub-condylar fracture which needed surgical interference in the form of open reduction and internal fixation. All the selected patients were informed about the surgical procedure, its potential hazards and possible complications. A written consent and an ethical clearance were obtained from each patient.

\section{Exclusion criteria:}

- Patients with old fractures.

- Patients with medical disease that may hinder normal healing.

\section{Patient grouping:}

The selected patients were divided into two groups, eight patients each;

\section{Group A:}

- The unilateral sub-condylar fractures were virtually reduced first using the digital 3D computed tomography (CT) through mirroring of the contra-lateral side by Iterative Closest Point algorithm (ICP).

- The fracture fixation was done using a computer manufactured patient-specific plate with an extended wing on the posterior border of the condylar neck to incase the two fractured segments allowing the auto-reduction of the fractured segments together into normal position. (Fig.1)

\section{Group B:}

- The unilateral sub-condylar fractures were manually reduced back into position and fixed in place by the standard trapezoidal mini-plate. (Fig.1)

\section{Preoperative assessment:}

Medical history and detailed history of the trauma was documented.

An accurate clinical examination was performed to detect the patients' complains of pain and tenderness, changes in dental occlusion, mouth opening restriction or deviation, or any other functional deficits.

A screening plain panoramic radiograph was performed to identify the post-traumatic fracture lines. Preoperative CT images were done to record the degree of fracture displacement and the condylar location. (Fig.2)



Fig. (1) Photographs showing the difference between the two plates used, which is the extended wing. 



Fig. (2) Preoperative CT radiographs showing the fracture line and the displaced condyle.

\section{Virtual fracture reduction and Processing of the} PS-condylar plate:

The aligned intact side served as a template to reposition the displaced fragments. It was automatically mirrored to the fractured side relying on the standard orientation of the unaffected regions of the skull around the fracture.

Computer modeling of the patient specific condylar plate was then performed. Each titanium plate was manufactured via direct metal laser sintering (DMLS). (Fig. 3)

\section{Operative procedures}

Standard retro-mandibular approach was performed for all cases under general anesthesia to reach the condylar fracture.
In group A patients, the segments were reduced together in normal position once the condylar segment was captured into the postero-medial wing of the plate. Auto-reduction of the segments was achieved. (Fig. 4)

While in group $B$ patients, manual reduction of the two segments was done and application of the trapezoidal plate in place was performed. In this group, some cases needed intra-operative Maxillo-Mandibular fixation to help in the reduction process. (Fig. 5)

Fixation of the plate holes on the segments was then performed using 2.0 mini-screws. Intra-operative occlusion of the patients was checked \& confirmed to be satisfactory. Closure was performed in layers and suture removal was done after one week. All patients were closely monitored postoperatively.

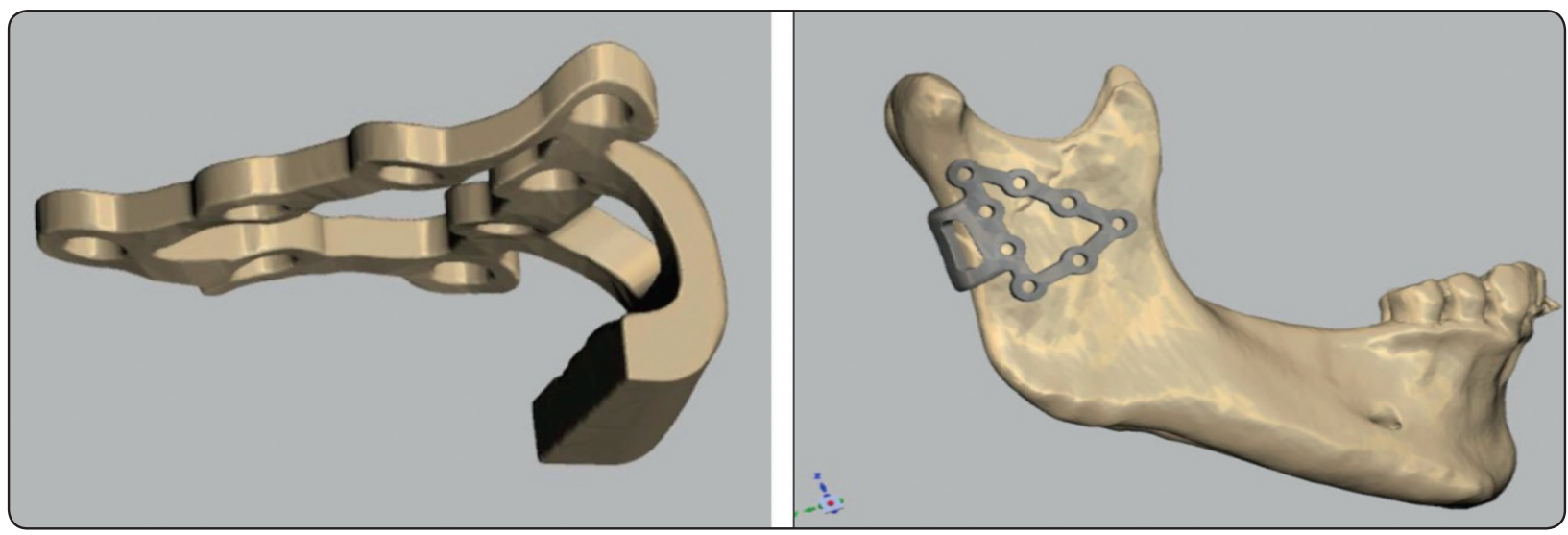

Fig. (3) Images showing the modeling of the patient specific condylar plate on the virtually reduced fracture. 


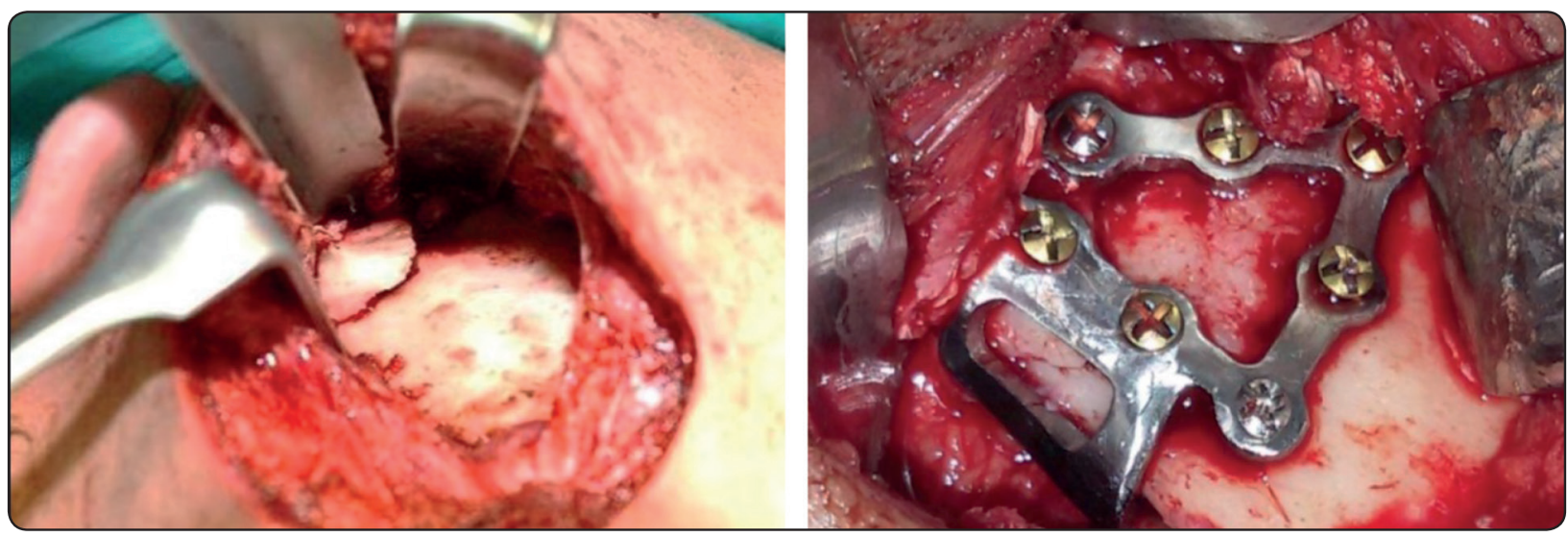

Fig. (4) Intra-operative photographs showing the exposure of the subcondylar fracture and its fixation by the PS-condylar plate

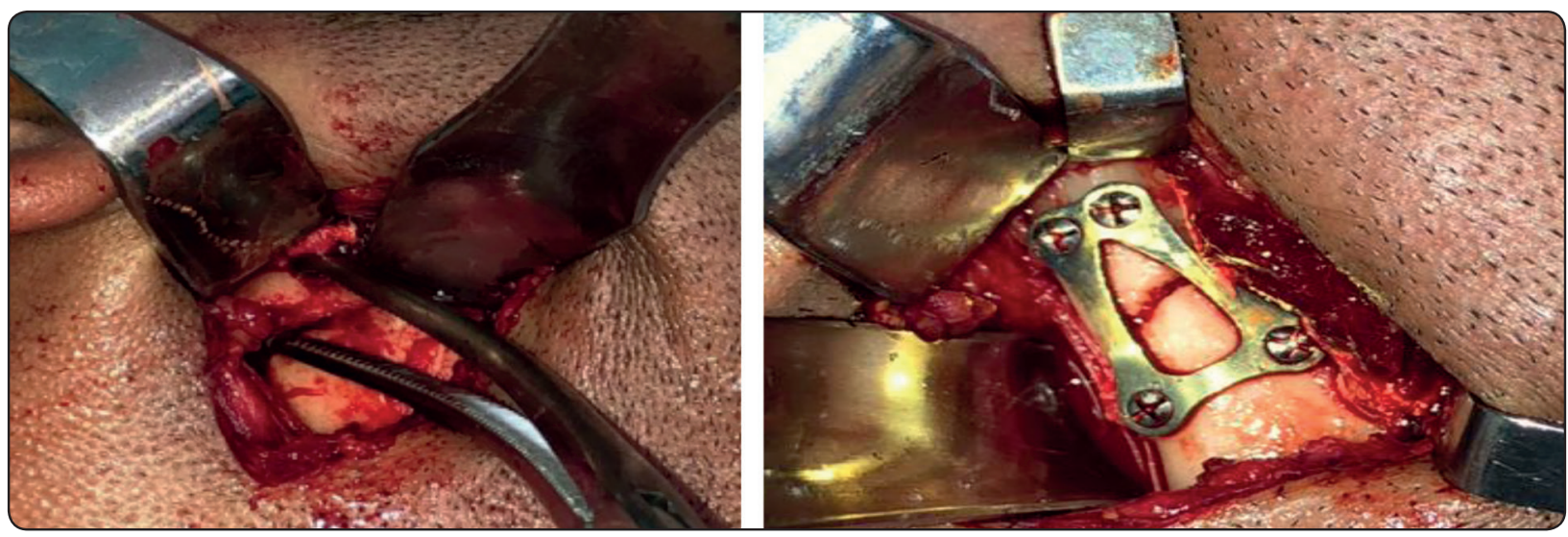

Fig. (5) Intra-operative photographs showing the manual reduction of the subcondylar fracture and its fixation by the standard trapezoidal plate

\section{Postoperative assessment}

Follow up of the patients continued for 3 months. Wounds were inspected for any signs of infection or dehiscence.

The clinical evaluation included the assessment of the occlusion, Maximum inter-incisal opening (MIO) and mandibular deviation at one week, 1 month \& 3 months.

Immediate postoperative $\mathrm{CT}$ was done to validate and compare the accuracy of the achieved postoperative fixation in both groups. This was done through the superimposition of the postoperative CT data on the preoperative virtually reduced 3D mandibular model as a reference CT scan data.

\section{RESULTS}

A male prevalence in this study was found with a mean age of 42 years. Other maxillofacial fractures were detected in some patients irrelevant to the fracture under study.

All surgeries were uneventful and the healing in all cases was normal with no evidence of surgical infection or tissue inflammation. Only normal postoperative swelling and pain were detected which resolved in the first week. No facial nerve injury was reported in any of the patients

Despite of the evident preoperative malocclusion in all patients, slight immediate postoperative occlusal discrepancy was found in both groups. 
However, postoperative occlusion in all patients was clinically acceptable at the end of the study interval. (Fig. 6).



Fig. (6) A postoperative photograph showing the clinical proper occlusion.

The preoperative limitation of mouth opening was significantly improved after fracture reduction and fixation at one week postoperatively. This continued in the form of a significant improvement in MIO throughout further study intervals with active and passive mouth exercises to reach a range of 39-47 $\mathrm{mm}$ at the end of the study. (Table 1)

TABLE (1): Showing mean values and SD of MIO for both groups.

\begin{tabular}{|c|c|c|c|c|c|}
\hline \multirow{2}{*}{ Period } & \multicolumn{2}{|c|}{ Group I } & \multicolumn{2}{c|}{ Group II } & \multirow{2}{*}{ P-value } \\
\cline { 2 - 5 } & Mean & SD & Mean & SD & \\
\hline Preoperative & 22 & 6.4 & 27.4 & 7.4 & 0.168 \\
\hline 1 week & 29.6 & 7.5 & 24.1 & 8.2 & 0.218 \\
\hline 1 month & 42.3 & 7 & 33.1 & 2.5 & 0.007 \\
\hline 3 months & 46.1 & 7.1 & 39.1 & 5.9 & 0.046 \\
\hline
\end{tabular}

Regarding the preoperative deviation on mouth opening, it was found to be reduced on week one postoperatively. However, at the end of the followup period, deviation was not completely absent in both groups.

The maximum operating time for reduction and fixation using the PS-condylar plate was 90 minutes with a mean of 75 minutes compared to a maximum of 120 minutes with a mean of 88 minutes using the standard reduction and trapezoid plate fixation technique

Radiographic CT scan revealed the almost equal accurate adaptation of both plates in both groups. The plates were found to be in the preoperative planned position. (Fig. 7-8)

In both groups, matching corresponding landmarks in the postoperative CT data after plate fixation fell almost precisely in place when compared to and superimposed on the preoperative virtually reduced fracture on the reference mandibular model. (Fig. 9-10)

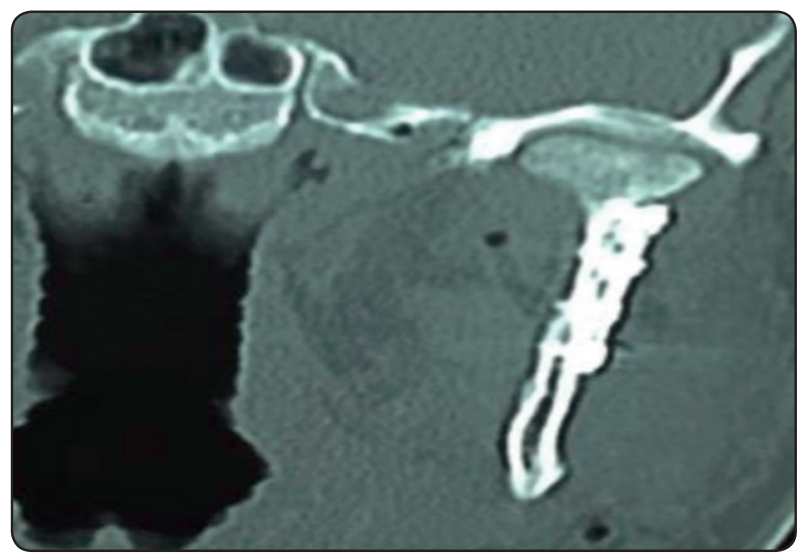

Fig. (7) CT image showing the reduced and fixed subcondylar fracture by the PS-condylar plate.

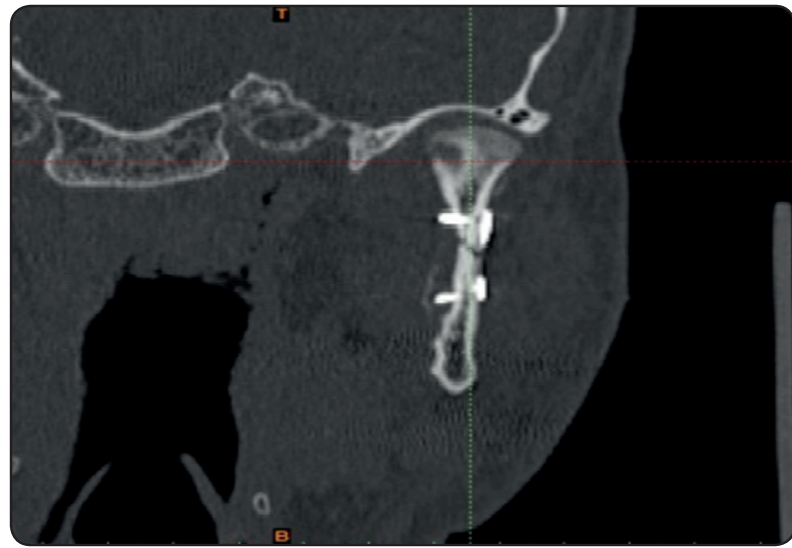

Fig. (8) CT image showing the reduced and fixed subcondylar fracture by the standard trapezoidal plate. 




Fig. (9) CT images showing the superimposition of the postoperative fixation by the PS-condylar plate (Blue color) over the preoperative virtually reduced 3D mandibular model (white color).

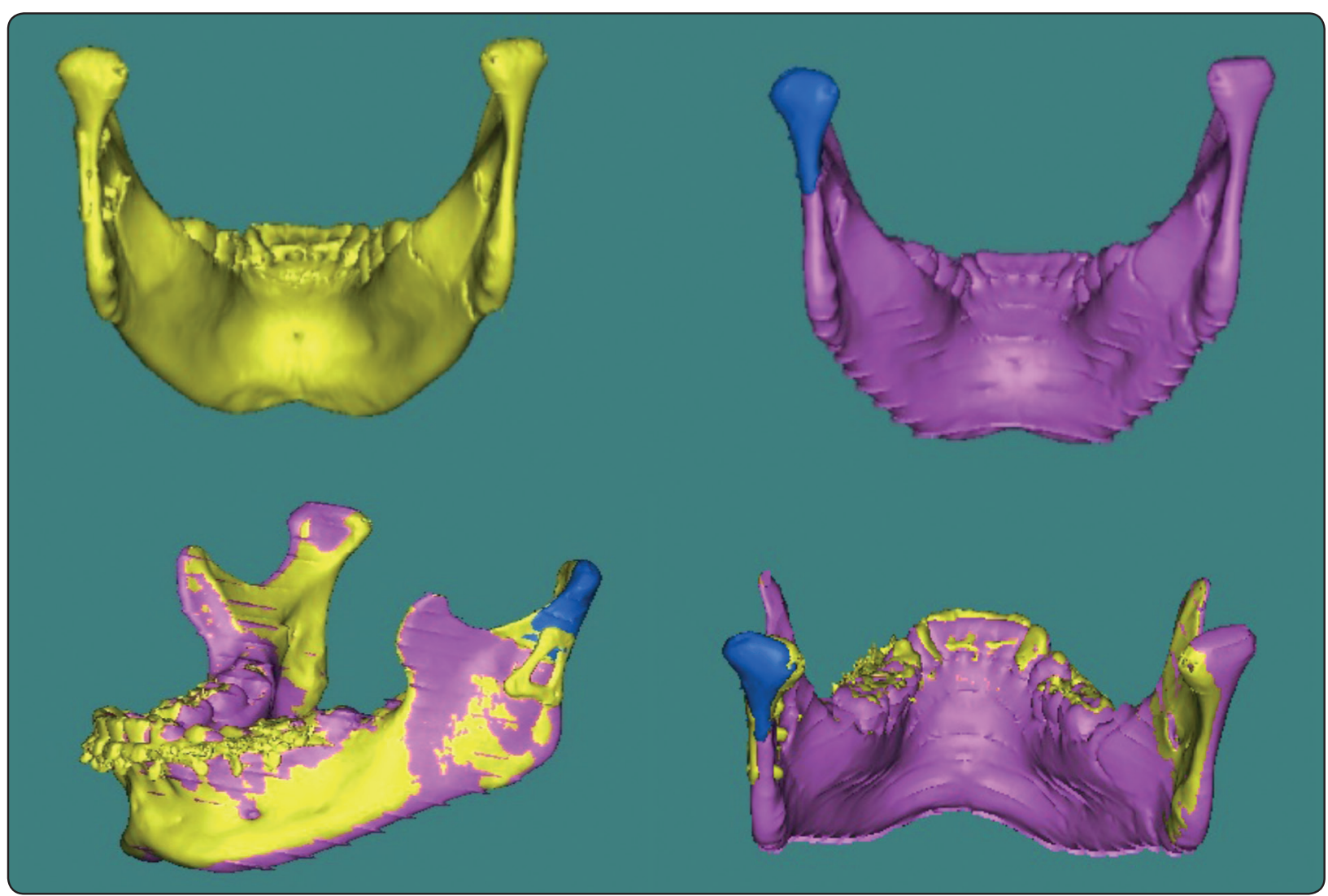

Fig. (10) CT images showing the superimposition of the postoperative fixation by the standard trapezoidal plate (yellow color) over the preoperative virtually reduced 3D mandibular model (purple color). 


\section{DISCUSSION}

Precise fracture reduction is crucial in order to restore the pre-injured anatomical geometry. Treatment of mandibular fractures using ORIF provided the advantage of direct visualization and reduction of the fracture line with simultaneous occlusion correlation $^{(11-12)}$.

Standard retro-mandibular approach was performed for all cases in this study as recommended by most surgeons ${ }^{(13)}$.

All of the surgeries were uneventful. The included patients experienced only postoperative swelling and pain which resolved in the first week. Consistent with other studies, this was considered a normal reaction to the surgical interference after the open reduction at the fracture site ${ }^{(14-16)}$.

Several experimental studies have been conveyed in the literature to assess the efficacy of various fixation methods in relation to functional stresses on the mandibular condyle. There is still no agreement on the optimal fixation method for subcondylar fractures ${ }^{(8,17-18)}$.

The stability of fixation and the regain of the normal function are important in evaluation of the management technique. This is complemented with the easiest technical application involved, the economic use of time and the minimal trauma resulting from the used surgical approach ${ }^{(19)}$.

For these reasons, recent studies highly support the digital software in surgery planning and custom plate manufacturing. Accordingly, in this study, a patient-specific auto-reducible plate was designed and manufactured in attempt to reach a simpler accurate maneuver for subcondylar fracture reduction and fixation compared to the standard trapezoid plate.

Voss et al ${ }^{(10)}$ used the preoperative CT (DICOM files) for virtual fracture reduction and computerized preoperative planning of the surgery. Similarly, we used the aligned intact side as a mirrored template to virtually reposition the displaced fragments.
The PS condylar plate customization and manufacturing for every patient were processed as described by Leiggener et al ${ }^{(20)}$. However, in this study, this plate contained a postero-medially extended wing at its posterior surface to wrap the posterior border of the condylar neck which provided precise auto-fracture reduction with adequate fixation of the subcondylar region. This provided a perfect adaptation at the fracture line between the condylar process and the ramus segment.

In this study, regarding the reduction and fixation, insignificant discrepancy was found between both groups. This was detected in the matching corresponding landmarks in the postoperative CT data after plate fixation which fell almost precisely in place when superimposed on the preoperative virtually reduced fracture on the reference mandibular model. This was due to the accurate fracture reduction under the intra-operative direct visualization of the fractured segments.

Slight immediate postoperative occlusal discrepancy was found in both groups, which was successfully self-corrected over the followup period. This was due to the utilized semi-rigid fixation coupled with the adaptation action of the oro-facial musculature.

This was consistent with Patil et al (21) who declared satisfactory occlusion in all patients. However, their study showed no deviation of mandible during maximum mouth opening contradicting our results in which deviation was not completely absent in both of our groups at the end of our follow-up period. This may be attributed to the initial different displacement range of the condyle in each patient after fracture and the subsequent muscle disorientation.

Lachner et al (22) and Jensen et al (23) reported that all patients returned to within normal range of opening ( 37 to $43 \mathrm{~mm}$ ) within 8 weeks. Similarly, in our study, the patients of both groups enjoyed an acceptable range of mandibular movements with significant improvement under active and passive mouth exercises to reach a range of $39-47 \mathrm{~mm}$ at the 
end of the study with minimal difference between both groups.

A crucial and significant difference in the operating time between the two groups was found in our study. The PS condylar plate seemed to offer faster application and easier fixation technique. This was due to the advantageous auto-reduction feature of the PS plate over the manually reduced segments of the standard trapezoid plate along with the eliminated possibility of the MMF use with the PS plate.

For these reasons, the easier technical application of the new PS condylar plate proved beneficial superseding the standard trapezoid plate with more acknowledged outcome.

\section{CONCLUSION AND SIGNIFICANCE}

Nowadays, in maxillofacial trauma, the merging of computer programs in surgery planning and patient specific plate designing and manufacturing is highly crucial due to its great benefits and accuracy.

The computer designed and manufactured PS auto-reducible plate proved to be highly beneficial in subcondylar fractures. The acknowledged fracture auto-reduction on plate application, the intra-operative time saving and the ease of plate application are highly demanded in any trauma related surgery.

The need to include biomechanical analysis on experimental mandibles is advisable to assess different stress bearing specifications of the different plates.

\section{REFERENCES}

1. Bhagol A, Singh V, Kumar I, Verma A. Prospective evaluation of a new classification system for the management of mandibular subcondylar fractures. J Oral Maxillofac Surg; 69(4):1159-65, 2011.

2. Villarreal PM, Monje F, Junquera LM, Mateo J, Morillo AJ, González C. Mandibular condyle fractures: determinants of treatment and outcome. J Oral Maxillofac Surg; 62(2):155-63, 2004.
3. Singh V, Bhagol A, Goel M, Kumar I, Verma A. Outcomes of open versus closed treatment of mandibular subcondylar fractures: a prospective randomized study. J Oral Maxillofac Surg; 68(6):1304-9, 2010.

4. Ellis, E. III, Throckmorton, G.S. Treatment of mandibular condylar process fractures: biological considerations. J Oral Maxillofac Surg; 63: 115- 134, 2005.

5. Oliveira R, Pagnoncelli R , and Nascimento D. The Treatment of Condylar Fractures: To Open or Not to Open? A Critical Review of this Controversy. International Journal of Medical Sciences; 5 (6): 313-18, 2008.

6. Richard H, Peterson G, and Goltz M. A Biomechanical Evaluation of Mandibular Condyle Fracture Plating Techniques. Journal of Oral and Maxillofacial Surgery: Official Journal of he American Association of Oral and Maxillofacial Surgeons; 60 (1): 73-80-1, 2002.

7. Choi B, Yi C and Yoo J. "Clinical Evaluation of 3 Types of Plate Osteosynthesis for Fixation of Condylar Neck Fractures." Journal of Oral and Maxillofacial Surgery; Official Journal of the American Association of Oral and Maxillofacial Surgeons 59 (7): 734-7, 2001.

8. Christophe M, Martin E, Kahn J and Zink S. Development and Biomechanical Testing of a New Osteosynthesis Plate (TCP) Designed to Stabilize Mandibular Condyle Fractures. Journal of Cranio-Maxillo-Facial Surgery; 35 (2): 84-90, 2008.

9. Markiewicz M and Bell R. The use of 3D imaging tools in facial plastic surgery. Facial plastic surgery clinics of North America; 19: 655, 2011.

10. Voss J, Varjas V, Raguse J, Thieme N, Richards R, Kamer L. Computed tomography-based virtual fracture reduction techniques in bi-mandibular fractures. J Cranio maxillofac Surg. 2016 Feb; 44(2):177 85

11. Hussein M, Gadala S, Khidr B: Evaluation of $3.2 \mathrm{~mm}$ stainless steel lag screw in the management of anterior mandibular fractures. Egyptian Dental Journal, 53: 16111616, 2007.

12. Schon, R., Gutwald R. and Schramm, A: Endoscopy assisted open treatment of condylar fractures of the mandible: Extraoral vs Intraoral approach. Int J Oral Mxillofac Surg; 31(3):237-43, 2002.

13. Jan K, Pohlenz P, Blessmann M, Blake F, Eichhorn W, Schmelzle R, and Heiland M. Clinical Follow-up Examination of Surgically Treated Fractures of the Condylar Process Using the Trans-parotid Approach. Journal of Oral and Maxillofacial Surgery; 68 (3): 611-17, 2010. 
14. Prabhu RK, Sinha R, Chowdhury SK, Chattopadhyay PK. Evaluation of facial nerve function following surgical approaches for maxillofacial trauma. Ann Maxillofac Surg; 2(1):36-40, 2012.

15. Bhutia O, Kumar L, Jose A, Roychoudhury A, Trikha A. Evaluation of facial nerve following open reduction and internal fixation of subcondylar fracture through retromandibular trans-parotid approach. Br J Oral Maxillofac Surg; 52(3):236-40, 2014.

16. Shi D, Patil PM, Gupta R. Facial nerve injuries associated with the retro-mandibular trans-parotid approach for reduction and fixation of mandibular condyle fractures. $\mathrm{J}$ Cranio maxillofac Surg; 43(3):402-7, 2015.

17. Parascandolo S, Spinzia A, Parascandolo S, Piombino P, Califano L. Two load sharing plate's fixation in mandibular condylar fractures: biomechanical basis. J Cranio maxillofac Surg.; 38(5):385-90, 2010.

18. Gealh WC, Costa JV, Ferreira GM, Iwaki Filho L. Comparative study of the mechanical resistance of 2 separate plates and 2 overlaid plates used in the fixation of the mandibular condyle: an in vitro study. J Oral Maxillofac Surg; 67(4):738-43, 2009
19. Airton V, Segundo L, Tharsila M, Oliveira D, Felipe E, Nogueira C, Carine A, and Rameiro F. Treatment of chronic condylar dislocation: comparison between two surgical techniques. Brazilian Journal of Surgery and Clinical Research BJSCR; 7 (3): 30-34, 2017.

20. Leiggener C, Messo E, Thor A, Zeilhofer HF and Hirsch JM. A selective laser sintering guide for transforming a virtual plan to real time surgery in composite mandibular reconstruction with free fibula osseous flaps, Int J Oral Maxillofac Surg; 38:187-92, 2009.

21. Patil, Ravi S, and Santosh S Gudi. Management of Subcondylar Fracture through Intraoral Approach with Rigid Internal Fixation.” Journal of Maxillofacial and Oral Surgery 10 (3): 209-15, 2011.

22. Lachner, J, J T Clanton, and P D Waite. Open Reduction and Internal Rigid Fixation of Subcondylar Fractures via an Intraoral Approach. Oral Surgery, Oral Medicine, and Oral Pathology 71 (3): 257-61, 1991.

23. Jensen T, Nørholt S, Dahl M, Hansen L, and Svensson P. Open Reduction and Rigid Internal Fixation of Mandibular Condylar Fractures by an Intraoral Approach: A LongTerm Follow-up Study of 15 Patients." Journal of Oral and Maxillofacial Surgery; 64 (12): 1771-79, 2006. 